\title{
Aggregation-Induced Emission Luminogen: The New Perspective in Photo-Degradation of Organic Pollution
}

Xing Feng, ${ }^{1}$ * Ying Li, ${ }^{2}$ Zhen Hu, ${ }^{1}$ Qingsong Wang, ${ }^{1}$ Mengsi Chen, ${ }^{1}$ Haoke Zhang, ${ }^{2}$ Miaomiao Kang, ${ }^{2}$ Zheng Zhao, ${ }^{2}$ Md. Monarul Islam, ${ }^{1}$ Jun Zhang, ${ }^{2}$ Ye Xiao, ${ }^{1}$ Menglong Zhang, ${ }^{1,3 *}$ Carl Redshaw, ${ }^{4}$ Qing Chen, ${ }^{5}$ Sheng Xie ${ }^{2 *}$ Jacky W. Y. Lam, ${ }^{2}$ Ben Zhong Tang ${ }^{2 *}$

${ }^{1}$ Guangdong Provincial Key Laboratory of Functional Soft Condensed Matter, School of Material and Energy, Guangdong University of Technology, Guangzhou 510006, P. R. China.

${ }^{2}$ Department of Chemistry, the Hong Kong Branch of Chinese National Engineering Research Center for Tissue Restoration and Reconstruction and Institute for Advanced Study, The Hong Kong University of Science and Technology Clear Water Bay, Kowloon, Hong Kong, P. R. China.

${ }^{3}$ Institute of Optoelectronic Materials and Technology, South China Normal University, Guangzhou 510631, P.R. China.

${ }^{4}$ Department of Chemistry \& Biochemistry, University of Hull, Cottingham Road, Hull, Yorkshire HU6 7RX, UK.

${ }^{5}$ Beijing enterprise water group limited, BEWG Mansion, T3, Poly International Plaza, 7th zone of Wangjing Dongyuan, Chaoyang District, Beijing, P. R. China.

*Correspondence: hyxhn@sina.com (X.F.)

**Correspondence: tangbenz@ust.hk (B. Z. T.) sheng.xie@ki.se (S.X.)

***Correspondence: mlzhang@m.scnu.edu.cn (M.Z) 


\begin{abstract}
:
Both the variety and uniqueness of organic semiconductors has contributed to the rapid development of environmental engineering applications and renewable fuel production, typified by photo-degradation of organic pollutants or water splitting. This paper presents a rare example of an aggregation-induced emission luminogen (AIEgen) as a highly efficient photo-catalyst for pollutant decomposition in an environmentally relevant application. Under irradiation, the tetraphenylethene-based AIEgen (TPE-Ca) exhibited high photo-degradation efficiency of up to $98.7 \%$ of Rhodaminein $(\mathrm{RhB})$ in aqueous solution. The possible photocatalytic mechanism was studied by electron paramagnetic resonance (EPR) and X-ray photoelectron spectroscopy (XPS) spectra, electrochemistry, thermal imaging technology, ultra-performance liquid chromatography and high-definition mass spectrometry (UPLC/HDMS), as well as by density functional theory (DFT) calculations. Cytotoxicity experiments indicated that the final photo-catalytic degradation products show biocompatibility. Among the many diverse AIEgens, this is the first AIEgen to be developed as a photo-catalyster of organic pollutants. This research will open up new avenues for AIEgens research, particularly for applications of environmental relevance.
\end{abstract}

Keywords: aggregation-induced emission, tetraphenylethene, photo-degradation, radical species, photo-catalysis mechanism 


\section{Introduction}

Increased energy consumption and environmental issues are becoming increasingly problematic as the global population grows and extensive industrialization continues at an ever increasing pace. ${ }^{[1]}$ Among them, water pollution is one of the most important environmental problems that mankind faces today. ${ }^{[2]}$ To-date, much effort have been devoted to exploring more efficient technologies for both prevention and control of water pollution, including the use of chemical and physical methods. Photocatalytic methods are one of the cutting-edge techniques available for water treatment because of mild operating conditions, high efficiency and relatively low cost. ${ }^{[3]}$

Currently, numerous photo-catalytically active materials including inorganic $\left(\mathrm{TiO}_{2}, \mathrm{ZnO}, \mathrm{CdS}\right.$ etc. $),{ }^{[4]}$ metal-free $\left(\mathrm{g}-\mathrm{C}_{3} \mathrm{~N}_{4}\right),{ }^{[5]}$ organometallic complexes ${ }^{[6]}$ and metal-nitrides $(\mathrm{GaN} / \mathrm{InGaN})^{[7]}$ as well as metal-organic frameworks (MOFs) ${ }^{[8]}$ have been extensively studied for pollutant decomposition, due to their good stability, high catalytic activity and favorable environmental characteristics. In the case of photo-catalytically active materials, they can capture photons with suitable light energy, then generate electrons and holes in the conduction and valence bands, respectively, both would react with water and oxygen to produce reactive oxygen species (ROS, such as hydroxyl radicals, superoxide, hydrogen peroxide and singlet oxygen) to then further degrade organic dyes or produce hydrogen. ${ }^{[9]}$ 
The ability to generate the ROS is a key point in the use of photoactive materials. ${ }^{[10]}$ Compared to the traditional photoactive materials mentioned above, organic semiconductor materials have attracted considerable attention as photo-catalysts, due to their tunable energy gap with a wide-range of visible-light low-cost and highly efficient utilization of solar energy. ${ }^{[11]}$ However, examples of photo-catalysts are rare due to the aggregation-caused quenching (ACQ) effect, which leads to low efficiency of ROS generation. ${ }^{[12]}$ On the other hand, the lack of catalytic reaction sites in many organic semiconductor materials lowers the photo-catalytic efficiency. The heterogeneous structures of alkalinized- $\mathrm{C}_{3} \mathrm{~N}_{4}, \mathrm{TiO}_{2}$-based hybrid materials ${ }^{[13]}$ can accelerate catalytic reactions by improving both the light harvesting ability and ROS generation efficiency via photo-generated electrons and holes.

In 2001, Tang's group developed a series of novel luminogens, defined as "aggregation-induced emission (AIE)", ${ }^{[12 a-c]}$ which exhibited an abnormal photo-physical phenomenon, viz weak or non-emissive in solution, but emitting bright light with high quantum yield (up to $100 \%$ ) in the aggregate state. These high-performance AIEgens with unique optical properties can be used for organic light-emitting diodes, fluorescence probes, as well as in cell imaging etc. ${ }^{[12]}$ On the other hand, AIEgens with appropriate molecular structures in the aggregated state are favorable for the formation of highly reactive oxygen species (ROS) for photodynamic therapy. ${ }^{[14]}$ Thus, if nascent ROS are involved in the photo-catalysis reaction of organic pollutants in water, then the AIEgen 
would be regarded as an excellent candidate for potential clean environmental applications. Previously, Tang et al. have demonstrated that a tailored cyanostilbene-based molecule with the AIE feature exhibited a controllable regio- and stereo-selective photo-dimerization or photo-cyclization reaction under UV irradiation with colour-tuneable properties in aqueous medium via a radical process. ${ }^{[15]}$ On the other hand, Zhao et al reported that cyanopyridinium-based cationic salts exhibited an enhanced emission with remarkable structure-dependent singlet-oxygen generation ability under white light illumination for photodynamic anticancer and antibacterial therapy. ${ }^{[16]}$ Both examples indicated that cyanostilbene derivatives possess a typical AIE characteristic with ideal photosensitizers for ROS generation. ${ }^{[17]}$

To explore the feasible use of AIEgens in environmental applications, this article presents the new perspective of AIEgens in photo-degradation of organic pollution in aqueous solution. The excellent organic photocatalyst of AIEgens plays a significant role to photo-degradation of Rhodamine with high efficiency (98.7\%), the whole process have been investigated by UV-vis spectroscopy, photoluminescence (PL), photo-electrochemistry and by ultra-performance liquid chromatography and high-definition mass spectrometry (UPLC/HDMS). More importantly, thermal imaging, electron paramagnetic resonance (EPR) spin-trap and X-ray photoelectron spectroscopic (XPS) techniques have been used to monitor the entire photo-catalytic process and to probe a possible mechanism. 


\section{Experimental section}

\subsection{Materials}

Unless otherwise stated, all reagents were purchased from commercial sources and used without further purification. Tetrahydrofuran was distilled prior to use. Dulbecco modified eagle medium (DMEM), fetal bovine serum (FBS), penicillin G $\left(100 \mathrm{U} \mathrm{mL}^{-1}\right)$, streptomycin $\left(100 \mathrm{U} \mathrm{mL}^{-1}\right)$, and $0.25 \%$ trypsin $0.53 \times 10^{-3} \mathrm{M}$ EDTA $\begin{array}{lllll}\text { solutionsolution } & \text { were } & \text { from } & \text { Gibco } & \text { (USA). }\end{array}$ 3-(4,5-dimethylthiazol-2-yl)-2,5-diphenyltetrazolium bromide (MTT) was from Sigma-Aldrich.

\section{2 synthesis}

\section{Synthesis of $\quad$ (Z)-2-(4'-(9H-carbazol-9-yl)-[1,1'-biphenyl]-4-yl)-3-}

\section{(4-(1,2,2-triphenylvinyl) phenyl) acrylonitrile (TPE-Ca):}

4-(1,2,2-triphenylvinyl)benzaldehyde $\quad$ (TPE-CHO) $\quad(627 \quad \mathrm{mg}, \quad 1.74$ mmol),2-(4'-(9H-carbazol-9-yl)- [1,1'-biphenyl]-4-yl) acetonitrile (1) (240 mg, 2.09 mmol) and potassium t-butoxide $(380 \mathrm{mg}, 2.09 \mathrm{mmol})$ were added into a $100 \mathrm{~mL}$ round-bottom flask under nitrogen asmosphere. Then $30 \mathrm{~mL}$ of ethanol was added. The mixture was refluxed overnight. After cooling to room temperature, the mixture was filtered and washed with ethanol three time. The residue was further crystallized in $\mathrm{CH}_{2} \mathrm{Cl}_{2}$ and hexane to give yellow powder (327 mg, yield $\left.70 \%\right) .{ }^{1} \mathrm{H}$ NMR (600 $\left.\mathrm{MHz}, \mathrm{CDCl}_{3}\right) \delta 8.16(\mathrm{~d}, J=7.8 \mathrm{~Hz}, 2 \mathrm{H}), 7.85(\mathrm{~d}, J=8.4 \mathrm{~Hz}, 2 \mathrm{H}), 7.77(\mathrm{q}, J=8.6 \mathrm{~Hz}$, 4H), $7.69(\mathrm{dd}, J=17.2,8.3 \mathrm{~Hz}, 4 \mathrm{H}), 7.53-7.46(\mathrm{~m}, 3 \mathrm{H}), 7.43(\mathrm{dd}, J=11.3,4.0 \mathrm{~Hz}$, 2H), $7.31(\mathrm{t}, J=7.4 \mathrm{~Hz}, 2 \mathrm{H}), 7.19-7.01(\mathrm{~m}, 17 \mathrm{H}) ;{ }^{13} \mathrm{C} \mathrm{NMR}\left(151 \mathrm{MHz}, \mathrm{CDCl}_{3}\right) \delta$ 
$146.64,143.35,143.31,143.18,142.42,141.77,140.85,140.77,140.01,138.99$, $137.40,133.99,131.98,131.61,131.38,131.34,131.30,128.84,128.42,127.93$, $127.88,127.70,127.65,127.45,126.93,126.75,126.72,126.49,126.02,123.49$, 120.37, 120.09, 118.12, 110.08, 109.81. HRMS FABS $\left(\mathrm{M}+\mathrm{H}^{+}\right)$calcd for $\mathrm{C}_{53} \mathrm{H}_{36} \mathrm{~N}_{2}$ 700.89, found 701.17 .

\subsection{X-ray Crystallography}

Crystallographic data of the compounds were collected on a Bruker APEX 2 CCD diffractometer with graphite monochromated Mo $\operatorname{K\alpha }$ radiation $(\lambda=0.71073 \AA)$ in the $\omega$ scan mode. The structure was solved by charge flipping or direct methods algorithms and refined by full-matrix least-squares methods on $F^{2}$. All esds (except the esd in the dihedral angle between two l.s. planes) were estimated using the full covariance matrix. The cell esdswere considered individually in the estimation of esds in distances, angles and torsion angles. Correlations between esds in cell parameters were only used when they were defined by crystal symmetry. An approximate (isotropic) treatment of cell esds was used for estimating esds involving l.s. planes. The final cell constants were determined through global refinement of the xyz centroids of the reflections harvested from the entire data set. Structure solution and refinement were carried out using the SHELXTL-PLUS software package. Data (excluding structure factors) on the structures reported here had been deposited with the Cambridge Crystallographic Data Centre with deposition numbers. CCDC 1914539 contain the supplementary crystallographic data for this paper. These data could be obtained free of charge from The Cambridge Crystallographic Data Centre 
via www.ccdc.cam.ac.uk/data request/cif.

\section{RESULTS AND DISCUSSION}

The synthetic route for TPE-Ca is shown in Scheme 1. A Knoevenagel reaction between 4-(1,2,2-triphenylvinyl) benzaldehyde and 2-(4'-(8a,9a-dihydro-9H-carbazol-9-yl)-[1,1'-biphenyl]-4-yl)acetonitrile in ethanol led to the formation of TPE-Ca (70\%). The target compound was fully characterized by ${ }^{1} \mathrm{H} /{ }^{13} \mathrm{C}$ NMR spectroscopy, single crystal X-ray diffraction, as well as by high resolution mass spectrometry (HRMS). TPE-Ca exhibits good solubility in common organic solvents, such as toluene, dichloromethane (DCM), tetrahydrofuran (THF), and 1,4-dioxane but does not dissolve in water.
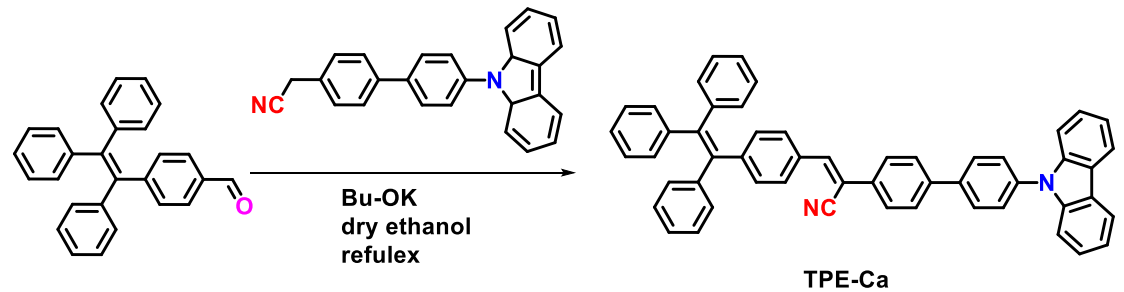

Scheme 1 Synthetic route for TPE-Ca

\subsection{X-ray single diffraction analysis}

A single crystal of TPE-Ca (CCDC:1914539) was grown from a mixture of $\mathrm{CH}_{2} \mathrm{Cl}_{2}$ and hexane (1:1) at room temperature. The single-crystal $\mathrm{X}$-ray structure of TPE-Ca adopts the monoclinic system with space group $\mathrm{P}_{1} 2 / \mathrm{n} 1$. The key parameter was listed in Table 1. The single crystal (Figure 1) adopts a twisted conformation with a large dihedral angle. The torsion angle between the terminal unit of the carbazole group and the phenyl ring is $70.61^{\circ}$. The molecular packing of TPE-Ca can best be described as off-set head-to-tail 
stacking. Each fragment of the molecule (such as the TPE and carbazole units) are linked to each other by several weak $\mathrm{C}-\mathrm{H} \cdots \pi$ interactions with distances in the range 2.28 to $2.80 \AA$. Generally, the non-planar conformation of TPE-Ca not only contributes to free intramolecular rotation in solution, but also to the formation of a close-packing matrix which can inhibit intramolecular rotation and result in enhanced fluorescence efficiency. Indeed, the TPE-Ca molecule exhibits clear AIE characteristics.

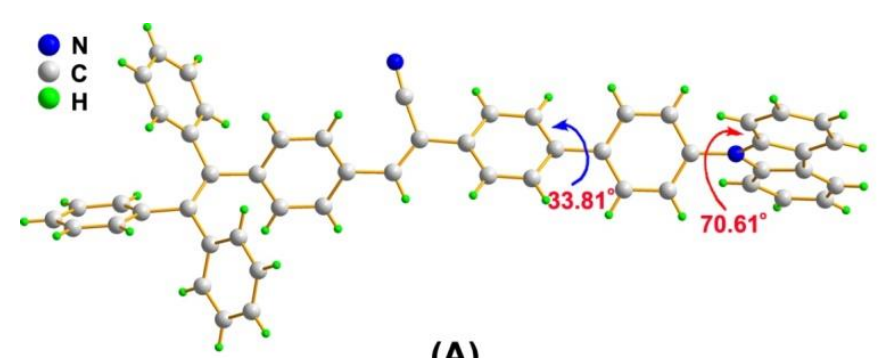

(A)

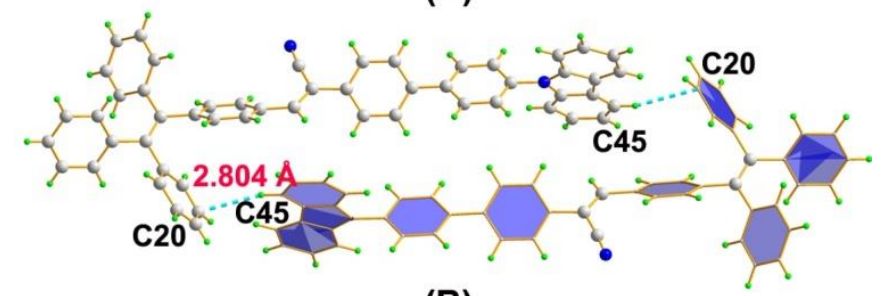

(B)

Figure 1 A) The X-ray structure of TPE-Ca and B) its crystal packing.

\subsection{Photophysical properties}

The photoluminescence (PL) spectra of TPE-Ca was measured in THF and in THF with different water fractions $\left(f_{\mathrm{w}}\right)$ (Figure 2). The TPE-Ca exhibited weak emission with a maximum emission peak at $505 \mathrm{~nm}$ in THF solution $\left(\Phi_{\mathrm{F}}\right.$ $=0.01)$. On gradual addition of water to the THF from 0 to $f_{\mathrm{w}}=99 \%$, the emission intensity of TPE-Ca enhanced ca. 100-fold with limited observed red-shifts $(<10 \mathrm{~nm})$ compared to the use of pure THF solution (Figure $2 \mathrm{~B})\left(\Phi_{\mathrm{F}}\right.$ 
$=0.36)$. Meanwhile, upon photo-excitation, TPE-Ca in the solid state displayed bright green emission at $502 \mathrm{~nm}$ with a narrow full width at half maxima $(\mathrm{FWHM})$. The fluorescence quantum yield $\left(\Phi_{\text {solid }}=0.6\right)$ is higher than that both in solution (0.01) and in aggregated state (0.36). On the other hand, the radiative decay rate $\left(k_{\mathrm{r}}\right)$ of TPE-Ca increased from $2 \times 10^{-9} \mathrm{~s}^{-1}$ in solution to $3.5 \times 10^{-8} \mathrm{~s}^{-1}$ in the solid state, while the non-radiative decay rate constant $\left(k_{\mathrm{nr}}\right)$ decreased from $19.8 \times 10^{-8} \mathrm{~s}^{-1}$ to $2.4 \times 10^{-8} \mathrm{~s}^{-1}$, indicating that the molecular rotation was restricted by several weak $\mathrm{C}-\mathrm{H} \cdots \pi$ interactions thereby blocking the non-radiative relaxation process. ${ }^{[18]}$ (Table 1)

Table 1. The photophysical and electrochemical properties of TPE-Ca..

\begin{tabular}{|c|c|c|c|c|c|c|c|c|c|}
\hline Compd. ${ }^{[a]}$ & $\begin{array}{c}\lambda_{\text {maxabs }} \\
(\mathrm{nm})\end{array}$ & $\begin{array}{r}\lambda_{\operatorname{maxPL}} \\
(\mathrm{nm})\end{array}$ & $\Phi_{f}$ & $\mathrm{t}(\mathrm{ns})$ & $\begin{array}{c}\mathrm{K}_{\mathrm{r}}\left(\times 10^{-8} \mathrm{~s}^{-1}\right) \\
\left(\Phi_{f} / \tau_{f}\right)\end{array}$ & $\begin{array}{c}\mathrm{K}_{\mathrm{nr}}\left(\times 10^{-8} \mathrm{~s}^{-1}\right) \\
\left(1 / \tau_{f}-\mathrm{k}_{\mathrm{r}}\right)\end{array}$ & $\begin{array}{c}\text { LUMO } \\
(\mathrm{eV})\end{array}$ & $\begin{array}{c}\text { HOMO } \\
(\mathrm{eV})\end{array}$ & $\begin{array}{l}\triangle \mathrm{E} \\
(\mathrm{eV})\end{array}$ \\
\hline \multirow{4}{*}{ TPE-Ca } & 378 & 505(THF) & 0.01 & 0.5 & 0.2 & 19.8 & & & \\
\hline & (THF) & $515(90 \%)$ & 0.24 & 1.5 & 1.6 & 5.1 & $-2.48^{[b]}$ & $-5.58^{[b]}$ & $3.10^{[\mathrm{b}]}$ \\
\hline & 414 & $514(99 \%)$ & 0.36 & 2.17 & 1.7 & 2.9 & $-2.19^{[\mathrm{cc}]}$ & $-5.00^{[\mathrm{c}]}$ & $2.81^{[\mathrm{d} d}$ \\
\hline & (film) & 504(film) & 0.6 & 1.7 & 3.5 & 2.4 & & & \\
\hline
\end{tabular}

[a] Measured in THF and THF/water mixtures with different water fractions at room temperature;[b] DFT/B3LYP/6-31G (d p)* using Gaussian 09, [c] Values calculated using the ferrocene HOMO level by Cyclic Voltammetry using the empirical formulae $\mathrm{HOMO}=-\left(4.8+\mathrm{E}_{\text {onset }^{\mathrm{ox}}}-\mathrm{E}_{\text {onset }(\mathrm{Fc})^{\mathrm{ox}}}\right)$. [d] Calculated from $\lambda_{\text {edge. }}$ 


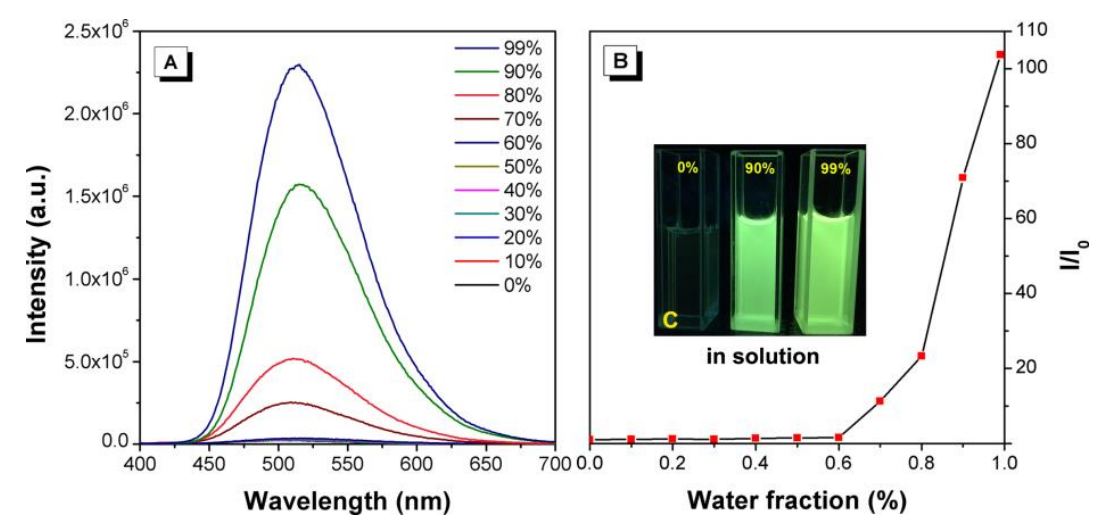

Figure 2. (A) PL spectra of TPE-Ca in THF/water mixtures with different water fractions $\left(f_{\mathrm{w}}\right)$, (B) Plot of relative $\mathrm{PL}$ intensity $\left(I / I_{0}\right)$ versus the composition of THF/water mixture of TPE-Ca, where $I_{0}$ is the PL intensity in pure THF solution. (C) Inset: fluorescent photographs of TPE-Ca in THF/ water mixtures $\left(f_{\mathrm{w}}=0,90 \%\right.$ and $\left.99 \%\right)$ taken under UV illumination $\left(\lambda_{\mathrm{ex}}=\right.$ $365 \mathrm{~nm})$.

\subsection{Detect radical}

According to previous reports, many examples have illustrated that AIEgens can be utilized to monitor photodynamic therapy (PDT), cell apoptosis, as well as the imaging of cell organelle by ROS generation under light irradiation, and these are processes are closely reliant on the active radical. ${ }^{[19]}$ Liu et al. reported the relationship between the value of the lowest singlet excited state (S1), the lowest triplet excited state $(\mathrm{T} 1)\left(\Delta \mathrm{E}_{\mathrm{S} 1-\mathrm{T} 1}, \Delta \mathrm{E}_{\mathrm{ST}}\right)$ and the efficiency to generate ROS by intersystem crossing (ISC); lower $\Delta \mathrm{E}_{\mathrm{ST}}$ values for AIEgens benefit the release of ROS. ${ }^{[20]}$ Thus, in the case of TPE-Ca, the optimized molecular geometry and the electron-density distribution of HOMO and LUMO were calculated using Gaussian 09 software at the B3LYP/6-31G(d, g) basis level (Table S2). ${ }^{[21]}$ The calculated TD-DFT value for $\Delta \mathrm{E}_{S T}$ is $0.26 \mathrm{eV}$, indicating that the TPE-Ca would be highly 
efficient for ROS generation and for the potential photo-degradation of organic pollutants.

To investigate the formation of ROS from TPE-Ca, The generation of $\bullet \mathrm{OH}$ species was further confirmed using the fluorescent probe, 4-hydroxyphenyl fluorescein $(\mathrm{HPF}),{ }^{[22]}$ which showed no emission but fluoresced strongly after reacting with the $\cdot \mathrm{OH}$ radical. As shown in Figure $3 \mathrm{~A}-\mathrm{B}$, upon illumination of the mixture of TPE-Ca and HPF in water, the fluorescence was greatly enhanced with a positive linear relationship between the irradiation time and fluorescence intensity. Both results were consistent with the origin of the $\cdot \mathrm{OH}$ signal being water in the presence of the TPE-Ca under irradiation.
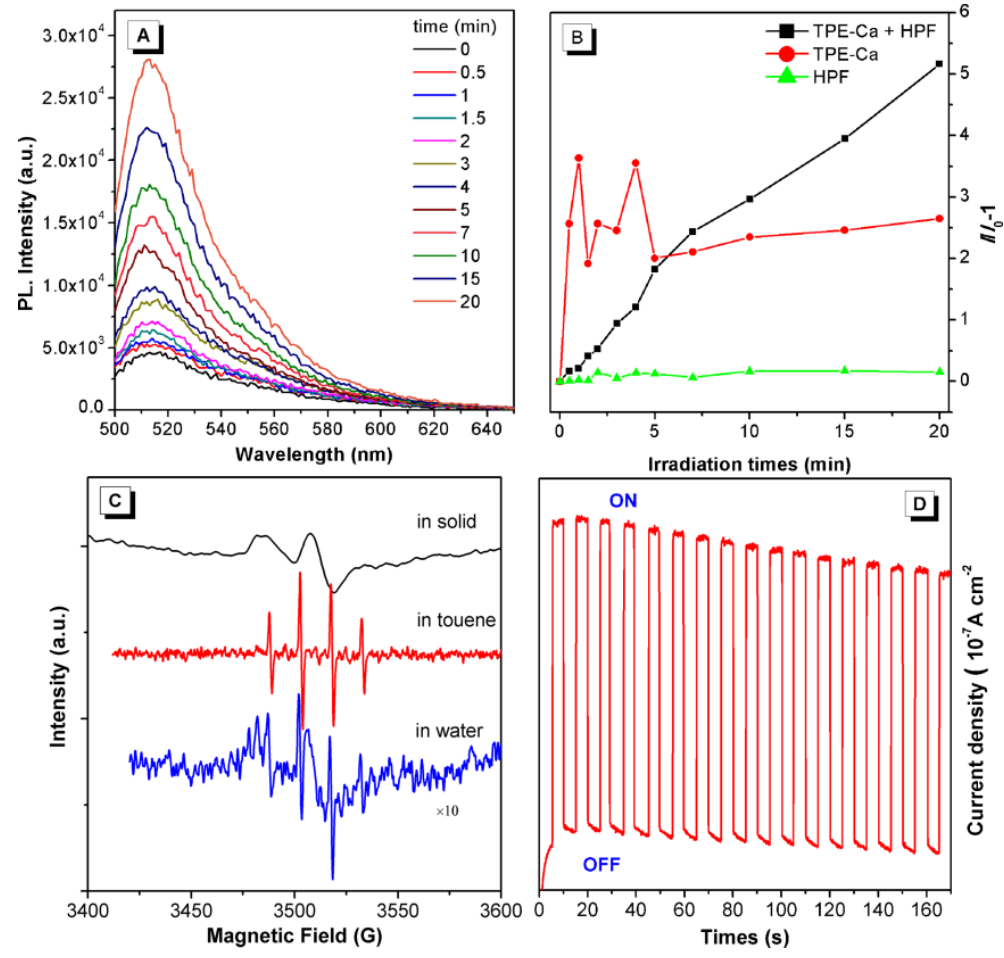

Figure 3. EPR spectra of TPE-Ca in solid state, in toluene and in water; Fluorescene spectrum of (B) $\bullet \mathrm{OH}$ dependent fluorescence response $\left(\lambda_{\mathrm{ex}}=490\right.$ $\mathrm{nm})$ of $\mathrm{HPF}(10 \mu \mathrm{M})$ under irradiation from 0 to $20 \mathrm{mins}$, (C) the linear relationship between irradiation time and fluorescence intensity, (D) Transient 
photocurrent response of pure TPE-Ca photocatalysts electrodes with light on-off cycles $\left[\mathrm{Na}_{2} \mathrm{SO}_{4}\right]=0.1 \mathrm{M}$ under solar light irradiation

More importantly, EPR spin-trap tests were performed for further confirming the $\cdot \mathrm{OH}$ radical in the solid state and on a suspension. As depicted in Figure $3 \mathrm{C}$, the EPR spectra of the solid sample TPE-Ca exhibited a strong EPR single with a $\mathrm{g}$ factor of 2.002 , which corresponds to the value for the free electron. ${ }^{[23]}$ Irradiating the TPE-Ca over 5 min using DMPO as spin-trapping reagent, a clear EPR $\bullet \mathrm{OH}$ signal with four well-resolved lines was detected centred at $\mathrm{g}=$ 2.006 in toluene, while two EPR signals were observed with g values of 2.008 and 2.015, respectively in water, due to the free electrons of TPE-Ca and $\bullet \mathrm{OH}$ species. Thus, we assume that the origin of the $\bullet \mathrm{OH}$ signal is generated from the $\mathrm{H}_{2} \mathrm{O}$ in the presence of radical of TPE-Ca, which would be further employed to photocatalytic photodegradation of organic dyes.

Furthermore, to test the separation efficiency of the photo-generated electron-hole pairs, the current versus time (I-t) characteristic was measured under a repeated ON/OFF illumination (Figure 3D). The TPE-Ca exhibited a clear photo response with stable photocurrent density under visible light irradiation. In the absence of light irradiation (OFF), the photocurrent rapidly dropped to almost zero, and the photocurrent reverted when the light was again ON. In addition, the current density decreased to $c a$. $10 \%$ under light irradiation after 20 cycles, which indicated that the TPE-Ca exhibited a reproducible photo-current with good photo-stability. 


\subsection{Photocatalytic activity}

To evaluate the photo-catalytic activity of TPE-Ca, the photo-degradation reaction was performed under visible light irradiation (simulated sunlight irradiation) in aqueous solution using rhodamine $\mathrm{B}(\mathrm{RhB})$ as the model organic pollutant. The adsorption equilibrium of the mixture of TPE-Ca (5mg) with $\mathrm{RhB}(10 \mathrm{mg} / \mathrm{L})$ were irradiated and the photo-degradation behaviour followed by UV-vis spectra.

Generally, the RhB aqueous solutions remain stable under visible light irradiation over 180 mins. $^{[24]}$ According to the Figure 4A, the UV-vis curves indicated that the $\mathrm{RhB}$ was almost completely degraded with the extreme degradation ratio of $98.7 \%$ within 120 mins in the presence of TPE-Ca, and the photo-degradation efficiency of TPE-Ca was higher than with common inorganic photo-catalysts, such as $\mathrm{C}_{3} \mathrm{~N}_{4},{ }^{[25]} \mathrm{TiO}_{2},{ }^{[26]}$ as well as some heterogeneous systems. ${ }^{[27]}$ On extending the irradiation time to $150 \mathrm{~min}$, we observed that the suspension colour changed from transparent to yellow-green, which may result from the formation of photo-catalytic degradation products that accelerate the solubility of TPE-Ca in aqueous solution. Clearly, the TPE-Ca can play a significant role in the photo-degradation of RhB. 

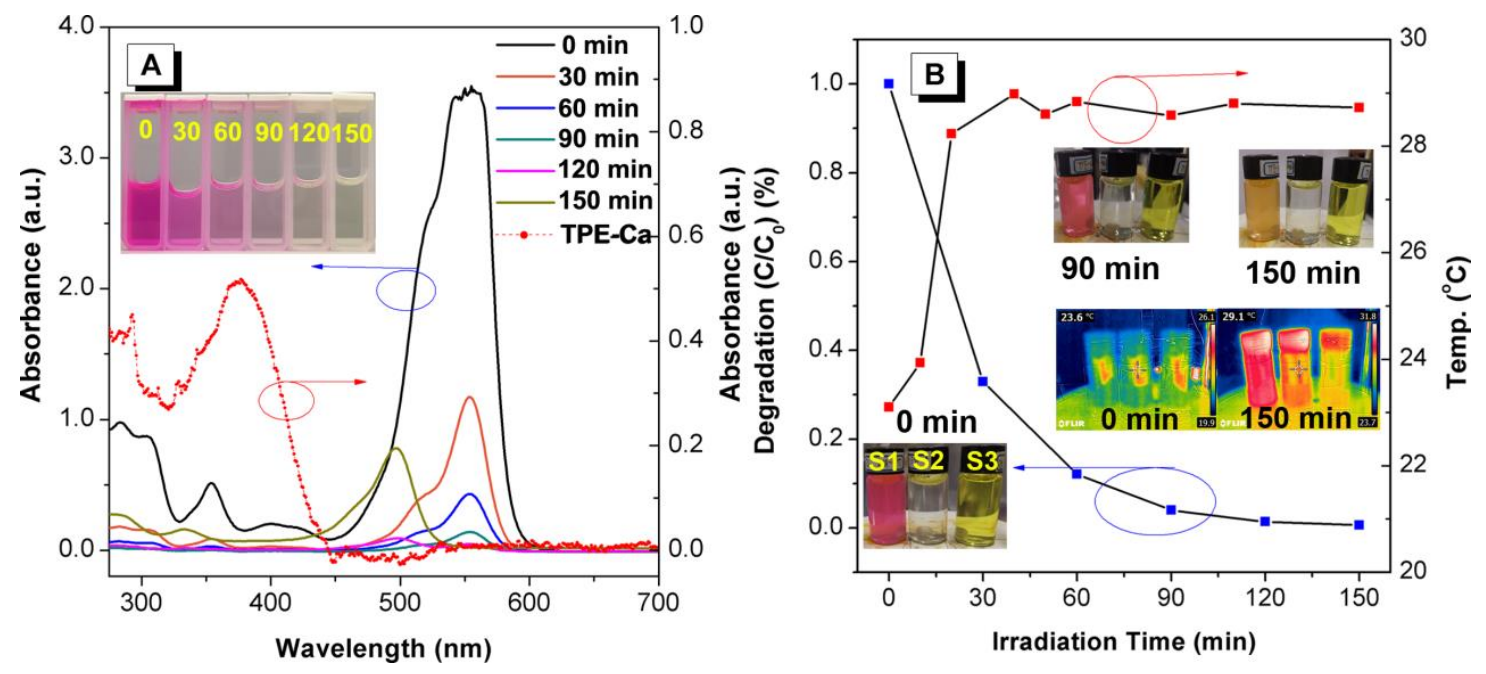

Figure 4. (A) UV-vis spectra to monitor the process of photo-catalytic degradation of $\mathrm{RhB}$, (B) Relative concentration $\left(\mathrm{C} / \mathrm{C}_{0}\right)$ and temperature versus time plot for the photo-degradation of $\mathrm{RhB}$ in the presence of TPE-Ca in aqueous solution. Insert: thermal imaging of samples (from left to right: S1: TPE-Ca@RhB in water, S2: TPE-Ca in water and S3: TPE-Ca in THF).

Meanwhile, thermal imaging technology was used to follow the entire photo-induced degradation process (Figure 4B). Interestingly, the change of temperature of the mixture TPE-Ca@RhB in water (S1) was remarkably different when compared to the TPE-Ca in water suspension (S2) and in THF (S3) solution when irradiated with sunlight. As Figure 4B shows, the temperature of TPE-Ca in water (S2) increased from $23.6{ }^{\circ} \mathrm{C}$ to $29.1{ }^{\circ} \mathrm{C}$ under light irradiation for $150 \mathrm{~min}$. More importantly, the temperature of S1 is higher than $\mathrm{S} 2$ which in turn is higher than S3. 


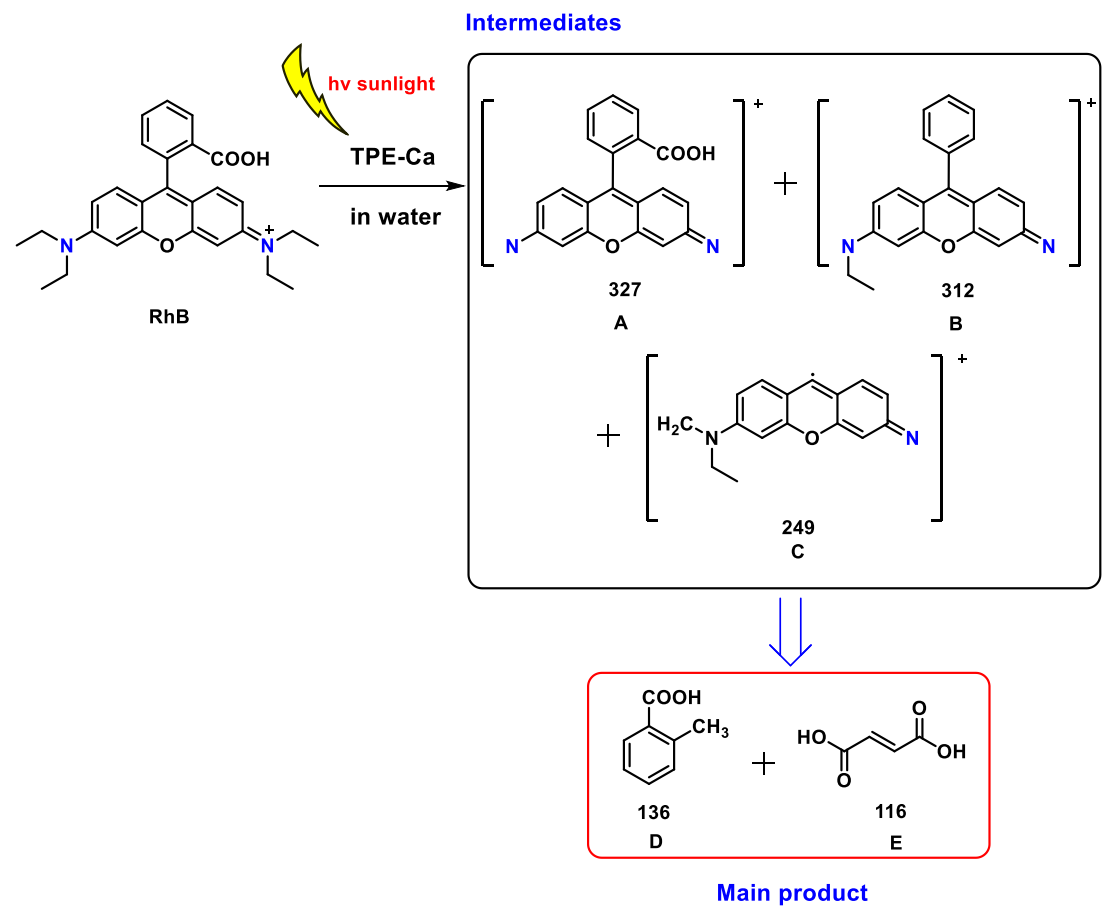

Figure 5. possible final products of the photo-catalytic degradation of $\mathrm{RhB}$ in the presence of TPE-Ca.

In addition, the final products of the photo-catalytic degradation of $\mathrm{RhB}$ were carefully examined by ultra-performance liquid chromatography and high-definition mass spectrometry (UPLC/HDMS). The UPLC chromatograms retention time at $3.45 \mathrm{~min}$ was observed, which corresponds to the intermediates A-E (Figure S8-13). The HDMS results indicate that the main intermediates contained mixtures of $\mathrm{N}$-de-ethylated intermediates (A, B and C), benzoic acid (D) and phthalic acid (E). Based on the UPLC/HDMS results, we propose a possible degradation pathway for $\mathrm{RhB}$ as listed in Figure 5. Firstly, after irradiation of TPE-Ca in water, the RhB was degraded to the intermediate radicals A, B and C. Subsequently, the unstable intermediates prefer to degrade to small molecules (D and E) under visible light irradiation. ${ }^{[25]}$ The HLF and COS7cells were incubated with final photo-catalytic degradation products 1 
(irradiation 120 mins) and 2 (irradiation $150 \mathrm{mins}$ ) in a medium for $1 \mathrm{~h}$ at $37^{\circ} \mathrm{C}$, and then washed three times with phosphate-buffered saline (PBS) buffer ( $\mathrm{pH}=$ 7.4) before imaging under a confocal fluorescence microscope. In Figure S15-17, the confocal images of HLF and COS7 cells exhibit normal morphology with good health, and both photodegradation finial compounds 1 and 2 seldomly enter the cells. In addition, the cell viability results showed that both cells remain close to $100 \%(>90 \%)$ over $24 \mathrm{~h}$ at a concentration of $0.6-3.0$ $\mathrm{g} / \mathrm{mL}$, indicating the low cytotoxicity of the degradation compounds.

\subsection{Photocatalytic Mechamism}

\subsubsection{XPS spectrum}

XPS was preformed to analyze the electronic and chemical environment of the radical and revealed that the detailed elements that TPE-Ca is comprised of, viz C1s (283.88 eV), N1s (398.7 eV) and O1s (540 eV) the Auger lines carbon KL1 $(1219 \mathrm{eV})$ and nitrogen KL2 (1139 eV) (Figure S18). The high-resolution XPS spectra of the C1s peaks can be divided into six peaks with binding energies at 283.35, 283.9, 284.95, 285.95, 288, $290.77 \mathrm{eV}$, which correspond to the $\mathrm{C}-\mathrm{C}, \mathrm{C}=\mathrm{C}, \mathrm{C}=\mathrm{N}, \mathrm{C} \equiv \mathrm{N}, \mathrm{C}=\mathrm{O}$ and $\pi$-plasmon, respectively. ${ }^{[29]}$ The N1s peak at 398.15, 399.11, 401.71 and 405.65 $\mathrm{eV}$ were assigned to $\mathrm{C}=\mathrm{N}, \mathrm{C} \equiv \mathrm{N}$ and the minor amount of oxidized $\mathrm{N}$ species $(\mathrm{N}-\mathrm{O}$. $(401.71 \mathrm{eV})$ and $\left.{ }^{+} \mathrm{N}=\mathrm{O}(405.65 \mathrm{eV})\right),{ }^{[30]}$ while the peaks at 531.25 and $532.54 \mathrm{eV}$ for O 1s were ascribed to $\mathrm{C}=\mathrm{O}, \mathrm{C}-\mathrm{O}-\mathrm{C}$, and the peaks at 533.5 and $534.7 \mathrm{eV}$ assigned to $\mathrm{N}-\mathrm{O}$ radical and $\mathrm{OH}$ groups from adsorbed water, respectively. ${ }^{[22,29]}$ (Table 2) The 
above analysis indicated that the radical is intact, which is fully consistent with the EPR results in the solid state.

Table 2. The high-resolution XPS spectrum of C1s, N1s and O1s peaks

\begin{tabular}{cccccc}
\hline Bond type & Peak BE & Bond type & Peak BE & Bond type & Peak BE \\
\hline C-C & 283.35 & $\mathrm{C}=\mathrm{N}$ & 398.15 & $\mathrm{C}=\mathrm{O}$ & 531.25 \\
$\mathrm{C}=\mathrm{C}$ & 283.9 & $\mathrm{CN}$ & 399.11 & $\mathrm{C}-\mathrm{O}-\mathrm{C}$ & 532.54 \\
$\mathrm{C}=\mathrm{N}$ & 284.95 & pyrrolic & 401.71 & $\mathrm{~N}-\mathrm{O}$ & 533.5 \\
$\mathrm{CN}$ & 285.95 & $\mathrm{~N}^{+}$ & 405.65 & $\mathrm{OH}$ & 534.7 \\
$\mathrm{C}=\mathrm{O}$ & 288 & & & & \\
$\pi$-plamson & 290.77 & & & & \\
\hline
\end{tabular}
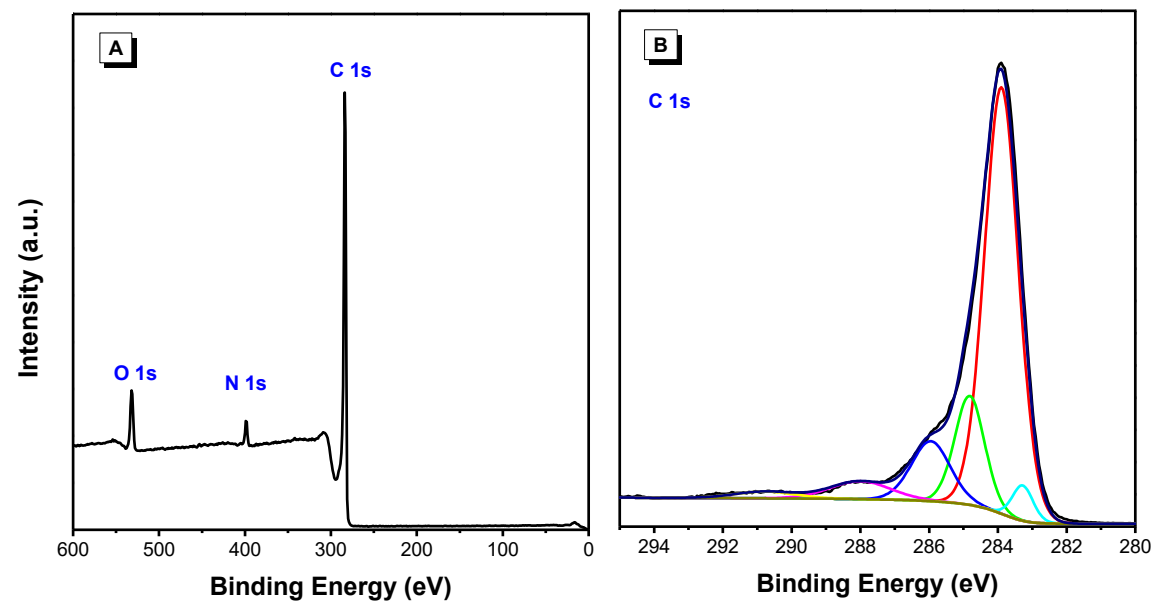

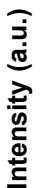
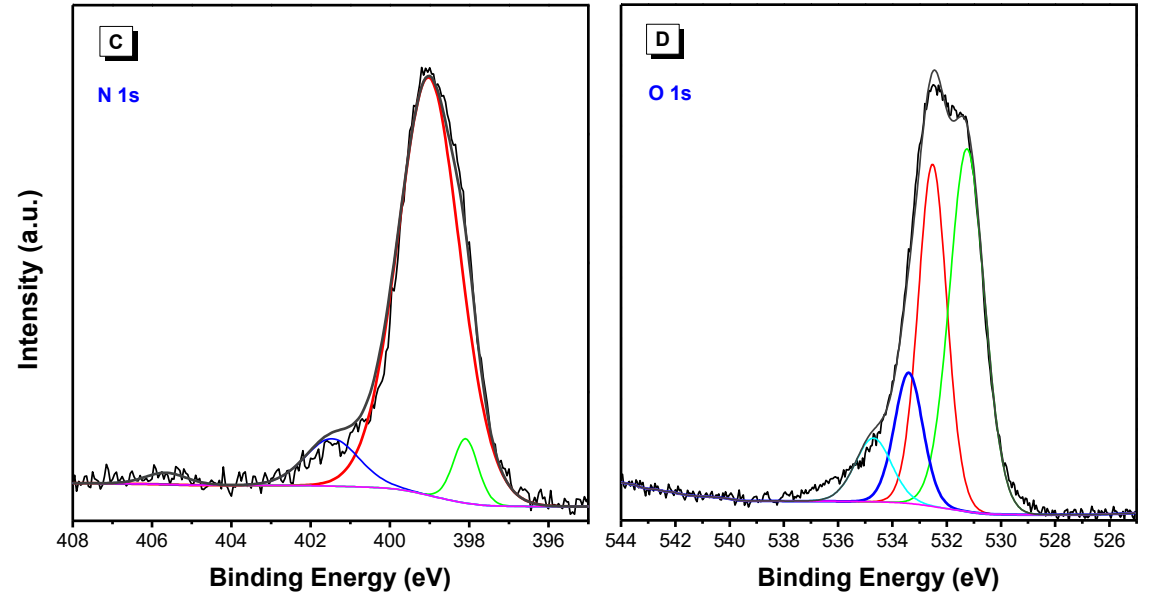

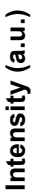

Figure 6. A) XPS spectrum of TPE-Ca; B) Carbon 1s, C) Nitrogen 1s and D) oxygen 1s XPS spectra of TPE-Ca. 


\subsubsection{Possible photocatalytic mechanism}

Thus, the observations from the EPR and XPS results illustrated the possible photocatalytic process: 1) A free electron of TPE-Ca was lost under irradiation resulting in radical TPE-Ca, 2) the radical can react with water to generate hydroxyl radical with an exothermic process by cleavage of the oxygen hydrogen bond and 3) the hydroxyl radical plays a significant role in degrading the $\mathrm{RhB}$ to achieve the final products (Figure 7). On the other hand, for S1, under irradiation, an exothermic reaction occurs when the radical of TPE-Ca reacts with $\mathrm{H}_{2} \mathrm{O}$ to achieve $\cdot \mathrm{OH}$. Moreover, the $\bullet \mathrm{OH}$ species involved in the oxidation reaction can generate enormous amounts of thermal energy, and S3 exhibited a relatively high temperature in comparison to $\mathrm{S} 2$, during the oxidation reactions indicating that the photo-degradation was rapid.
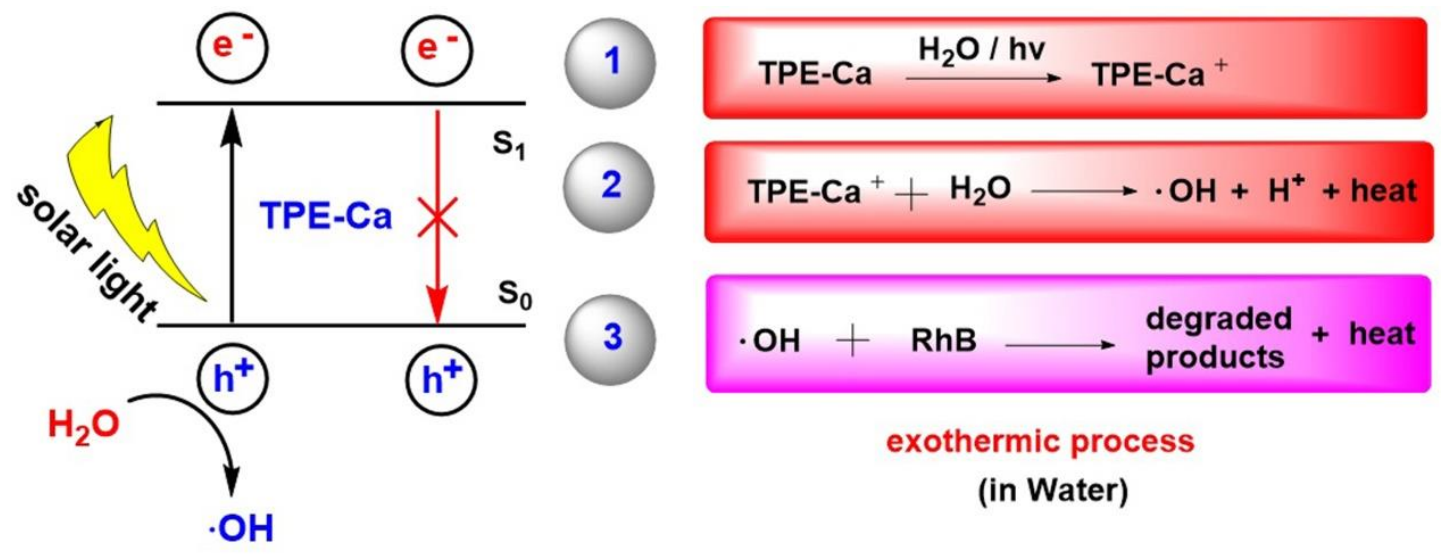

Figure 7. The possible photocatalytic process of TPE-Ca

\section{Conclusion}

In summary, we have explored an efficient photo-degradation method for organic pollutants $(\mathrm{RhB})$ by using a tetraphenylethene-based AIEgen (TPE-Ca) as a photo-catalyst. UV-vis spectroscopic monitoring of the TPE-Ca system 
revealed enhanced photo-catalytic ability towards photo-degradation of $\mathrm{RhB}$ which occurred with high efficiency $(98.7 \%)$ under white light irradiation over 120 min. Importantly, both chemical and physical interactions generate enormous amounts of thermal energy and enhance the local environmental temperature. The nature of the final degradation products were confirmed by ultra-performance liquid chromatography and high-definition mass spectrometry (UPLC/HDMS), and these products displayed weak cytotoxicity towards HLF and COS7 cell lines. A photo-catalytic mechanism was proposed on the basis of EPR and XPS, the results for which indicated that the TPE-Ca displayed a free electron signal, which would induce $\bullet \mathrm{OH}$ for further photo-catalytic processes. We report here the first example of an AIEgen as a high-performance photo-catalyst for environmental applications, and the use of thermal imaging technology for monitoring the photo-induced degradation process. This research opens up new avenues in the applications of AIE research.

\section{Acknowledgements}

This work was supported by and the National Natural Science Foundation of China (21602014, 51773020 and 21490574), “One Hundred Talents Program” and Guangdong provincial key laboratory of functional soft condensed matter of the Guangdong University of Technology (GDUT) (1108-220413205). M. M. Islam thanks to MOST, P. R. China for Talented Young Scientist Program (TYSP) Fellowship (402180111); CR thanks the EPSRC for an Overseas Travel Grant. We also thank the technical support from AIEgen Biotech Co., Ltd.. 


\section{Author contributions}

X. Feng and Y. Li contributed equally to this work.

\section{Competing interests}

The authors declare no competing financial interests.

\section{References}

[1] a) C. J. Vörösmarty, P. B. McIntyre, M. O. Gessner, D. Dudgeon, A. Prusevich, P. Green, S. Glidden, S. E. Bunn, C. A. Sullivan, C. Reidy Liermann \& P. M. Davies. Nature, 467 (2010), 555-561; b) H. Zhang, D. Chen, X. J. Lv, Y. Wang, H. X. Chang and J. H. Li, Environ. Sci. Technol. 44 (2010), 1107-1111.

[2] a) M. N. Chong, B. Jin, C. W. Chow, C. Saint, Water Res. 44 (2010), 2997-3027;

b) I. Michael, L. Rizzo, C. McArdell, C. Manaia, C. Merlin, T. Schwartz, C. Dagot, D. Fatta-Kassinos, Water Res. 47 (2013), 957-995.

[3] a) K. Wenderich, G. Mul, Chem. Rev. 116 (2016), 14587-14619; b) A. Kubacka, M. Fernández-García, G. Colón, Chem. Rev. 112 (2011), 1555-1614; (c) X. Chen, S. Shen, L. Guo, S. S. Mao, Chem. Rev. 110(2010), 6503-6570.

[4] a) J. Yan, P. Li, Y. Ji, H. Bian, Y. Li, S. Liu, J. Mater. Chem. A 5 (2017), 21478-21485; b) W. Wang, J. Dong, X. Ye, Y. Li, Y. Ma, L. Qi, Small 12 (2016), 1469-1478; c) M. Zhang, Robert W. Mitchell, H. Huang, R. E. Douthwaite, J. Mater. Chem. A 5 (2017), 22193-22198; d) N. Waiskopf, Y. Ben-Shahar, M. Galchenko, I. Carmel, G. Moshitzky, H. Soreq, U. Banin, Nano Lett. 16 (2016), 4266-4273. 
[5] a) A. Dhakshinamoorthy, A. M. Asiri, H. Garcia, Angew. Chem. Int. Ed. 55 (2016), 5414-5445; b) J. Qiu, X. Zhang, Y. Feng, X. Zhang, H. Wang, J. Yao, Appl.Catal. B-Environ. 231 (2018), 317-342; c) B. Zhang, S. Y. Zhao, H. 'H. Wang, T. J. Zhao, Y. X. Liu, L. B. Lv, X. Wei, X. H. Li, J. S. Chen, Chem. Commun. 53 (2017), 10544-10547; d) M. Zhou, Z. Hou, L. Zhang, Y. Liu, Q. Gao, X. Chen, Sustain. Energ. Fuels 1 (2017), 317-323; e) Qinghua Liang Zhi Li Xiaoliang Yu Zheng-Hong Huang Feiyu Kang Quan-Hong Yang, Adv. Mater. 27 (2015), 4634-4639.

[6] a) A. M. Sevim, J. Organomet. Chem. 832 (2017), 18-26; b) M. B. Tahir, J. Inorg. Organomet. P. 28 (2018), 2160-2168.

[7] S. Routray, T. R. Lenka, IEEE T. Nanotechnol. 17 (2018), 1118-1124.

[8] X. Liu, B. Liu, G. Li, Y. Liu, J. Mater. Chem. A 6 (2018), 17177-17185.

[9] H. Jia, W. He, W. G. Wamer, X. Han, B. Zhang, S. Zhang, Z. Zheng, Y. Xiang, J.-J. Yin, J. Phys. Chem. C 118 (2014), 21447-21456.

[10] a) N. Zhu, S. Wang, C. Tang, P. Duan, L. Yao, J. Tang, P. K. Wong, T. An, D.D. Dionysiou, Y. Wu, Environ. Sci. Technol. 53 (2019), 1585-1594; b) T. Ahmad, U. Farooq, R. Phul, Ind. Eng. Chem. Res. 57 (2018), 18-41; c) L. Zeng, X. Guo, C. He, C. Duan, ACS Catal. 6 (2016), 7935-7947; d) T. Montini, M. Melchionna, M. Monai, P. Fornasiero, Chem. Rev., 116 (2016), 5987-6041; e) T. H. Jeon, M. S. Koo, H. Kim, W. Choi, ACS Catal. 8 (2018), 11542-11563.

[11] a) L. Zhao, Y. L. Lin, H. Kim, N. C. Giebink, B. P. Rand, ACS Energy Lett. 3 (2018), 2708-2712; b) K. Rajeshwar, J. Phys. Chem. Lett. 2 (2011), 1301-1309. 
[12] a) J. Luo, Z. Xie, J. W. Y. Lam, L. Cheng, H. Chen, C. Qiu, H. S. Kwok,X. Zhan, Y. Liu, D. Zhu, B. Z. Tang, Chem. Commun. 18 (2001), 1740-1741; b) Y. Hong, J. W. Y. Lam, B. Z. Tang, Chem. Soc. Rev. 40 (2011), 5361-5388; c) J. Mei, N. L. C. Leung, R. T. K. Kwok, J. W. Y. Lam, B. Z. Tang, Chem. Rev. 115 (2015), 11718-11940. d) N. Sekkat, H. van den Bergh, T. Nyokong and N. Lange, Molecules 2012, 17, 98; e) W. A. Qureshi, Nat. Rev. Drug Discovery 3 (2004), 447-450; f) M. M. Islam, Z. Hu, Q. Wang, C. Redshaw, X. Feng, Mater. Chem. Front. 3 (2019), 762-781.

[13] a) Q. Liang, Z. Li, X. Yu, Z.-H. Huang, F. Kang, Q.-H. Yang, Adv. Mater. 27 (2015), 4634-4639; b) H. Zhu, N. Goswami, Q. Yao, T. Chen, Y. Liu, Q. Xu, D. Chen, J. Lu, J. Xie, J. Mater. Chem. A 6 (2018), 1102-1108; c) J. Chen, C.-L. Dong, D. Zhao, Y.-C. Huang, X. Wang, L. Samad, L. Dang, M. Shearer, S. Shen, L. Guo, Adv. Mater. 29 (2017), 1606198.

[14] a) S. Xu, W. Wu, X. Cai, C.-J. Zhang, Y. Yuan, J. Liang, G. Feng, P. Manghnania, B. Liu, Chem. Commun. 53 (2017), 8727-8730; b) C. Zhu, R. T. K. Kwok, J. W. Y. Lam, B. Z. Tang, ACS Appl. Bio. Mater. 1 (2018), 1768-1786.

[15] P. Wei, J.-X. Zhang, Z. Zhao, Y. Chen, X. He, M. Chen, J. Gong, H. H.-Y. Sung, I. D. Williams, J. W. Y. Lam, B. Z. Tang, J. Am. Chem. Soc. 140 (2018), 1966-1975.

[16] N. Zhao, P. Li, J. Zhuang, Y. Liu, Y. Xiao, R. Qin, N. Li, ACS Appl. Mater. Interfaces 11 (2019), 11227-11237; b) M. Chen, L. Li, H. Nie, J. Tong, L. Yan, B Xu, J. Z. Sun, W. Tian, Z. Zhao, A. Qin, B. Z. Tang, 6 
(2015), 1932-1937; c) M. Chen, R. Chen, Y. Shi, J. Wang, Y. Cheng, Y. Li, X. Gao, Y. Yan, J. Z. Sun, A. Qin, R. T. K. Kwok, J. W. Y. Lam, B. Z. Tang, Adv. Funct. Mater. 28 (2018), 1704689

[17] a) B.-K. An, J. Gierschner, S. Y. Park, Acc. Chem. Res. 45 (2011), 544-554; b) K. Kokado, K. Sada, Angew. Chem. Int. Ed. 2019, 10.1002/anie.201814462

[18] a) S. Sekiguchi, K. Kondo, Y. Sei, M. Akita, M. Yoshizawa, Angew. Chem. Int. Ed. 55 (2016), 6906-6910; b) Y. Hong, Y. Dong, H. Tong, Z. Li, M. Häußler, J. W. Y. Lam and B. Z. Tang, Proc., SPIE 6470, Organic Photonic Materials and Devices IX, 64700T, 2007; c) J. Wang, X. Gu, P. Zhang, X. Huang, X. Zheng, M. Chen, H. Feng, R. T. K. Kwok, J. W. Y. Lam, B. Z. Tang, J. Am. Chem. Soc., 139 (2017), 16974-16979.

[19] a) Z. Zheng, T. Zhang, H. Liu, Y. Chen, R. T. K. Kwok, C. Ma, P. Zhang, H. H. Y. Sung, I. D. Williams, J. W. Y. Lam, K. S. Wong, B. Z. Tang, ACS Nano 12 (2018), 8145-8159; b) J. Shi, Y. Li, Q. Li, Z. Li, ACS Appl. Mater. Interfaces 10 (2017), 12278-12294; c) J. Mei, Y. Huang, H. Tian, ACS Appl. Mater. Interfaces 10 (2018), 12217-12261; d) S. Chen, Q. Li, X. Wang, Y.-W. Yang, H. Gao, J. Mater. Chem. B 6 (2018), 5198-5214; e) F. Wu, X. Wu, Z. Duan, Y. Huang, X. Lou, F. Xia, 2019, small 10.1002/smll.201804839; f) Xingshu Li, Jihoon Kim, Juyoung Yoon, Xiaoyuan Chen, Adv. Mater. 29 (2017), 1606857; g) Ji Qi Chao Chen Dan Ding Ben Zhong Tang, Adv Healthc Mater. 7 (2018), 1800477.

[20] a) S. Xu, Y. Yuan, X. Cai, C.-J. Zhang, F. Hu, J. Liang, G. Zhang, D. Zhang, B. 
Liu, Chem. Sci., 6 (2015), 5824-5830; b) S. Xu, W. Wu, X. Cai, C.-J. Zhang, Y. Yuan, J. Liang, G. Feng, P. Manghnani, B. Liu, Chem. Commun. 53 (2017), 8727-8730.

[21] M. J,Frisch, G. W. Trucks, H. B. Schlegel, G. E. Scuseria, M. A. Robb, J. R. Cheeseman. Gaussian 09, revision A.02. Wallingford CT: Gaussian, Inc.; 2009.

[22] a) M.-D. Li, N.-K. Wong, J. Xiao, R. Zhu, L. Wu, S.-Y. Dai, F. Chen, G. Huang, L. Xu, X. Bai, M. R. Geraskina, A. H. Winter, X. Chen, Y. Liu, W. Fang, D. Yang, D. L. Phillips, J. Am. Chem. Soc. 140 (2018), 15957-15968; b) M. Garcia-Diaz, Y.-Y. Huang, M. R. Hamblin, Methods, 109 (2016), 158-166; c) Ki. R. Fitch, A. P. Goodwin, Chem. Mater. 26 (2014), 6771-6776.

[23] F. Gerson, W. Huber, Electron Spin Resonance Spectroscopy of Organic Radicals, WILEY-VCH Verlag GmbH \& Co. KGaA, Weinheim, 2003.

[24] S. Tonda, S. Kumar, S. Kandula, V. Shanker. J. Mater. Chem. A 2 (2014), $6772-6780$.

[25] Q. Liang, Z. Li , X. Yu , Z.-H. Huang, F. Kang, Q.-H. Yang, Adv. Mater. 27 (2015), 4634-4639.

[26] Z. Zhao, G. Liu, B. Li, L. Guo, C. Fei, Y. Wang, L. Lv, X. Liu, J. Tian, G. Cao, J. Mater. Chem. A 3 (2015), 11320-11329.

[27] K. Maeda, K. Domen, Angew. Chem. Int. Ed. 51 (2012), 9865-9869.

[28] T. Xu, D. Wang, L. Dong, H. Shen, W. Lu, W. Chen, Appl.Catal. B-Environ. 244 (2019), 96-106.

[29] a) G. Chen, X. Wang, J. Li, W. Hou, Y. Zhou, J. Wang, ACS Appl. Mater. 
Interfaces 7 (2015), 18508-18518; b) F. Ciccullo, N. M. Gallagher, O. Geladari, Chassé, A. Rajca, M. B. Casu, ACS Appl. Mater. Interfaces 8 (2016), 1805-1812; c) S. Zhao, M. Lan, X. Zhu, H. Xue, T.-W. Ng, X. Meng, C.-S. Lee, P. Wang, W. Zhang, ACS Appl. Mater. Interfaces 7 (2015), 17054-17060; d) J. F. Moulder, W. F. Stickle, P. E. Sobol, K. D. Bomben, Handbook of X-ray Photoelectron Spectroscopy, Physical Electronics, Inc. Eden Prairie, Minnesota, USA, 1995. [30] a) B. Gadgil, P. Damlin, A. Viinikanoja, M. Heinonen, C. Kvarnstrőm, J. Mater. Chem. A 3 (2015), 9731-9737; b) Y. Lin, D. Su, ACS Nano 8 (2014), 7823-7833; c) P. Yang, R. Wang, M. Zhou, X. Wang, Angew. Chem. Int. Ed. 57 (2018), 8674-8677; d) J. Z. Low, G. Kladnik, L. L. Patera, S. Sokolov, G. Lovat, E. Kumarasamy, J. Repp, L. M. Campos, D. Cvetko, A. Morgante, L. Venkataraman, Nano Lett. 19 (2019), 2543-2548; e) K. Flavin, K. Lawrence, J. Bartelmess, M. Tasior, C. Navio, C. Bittencourt, D. F. O’Shea, D. M. Guldi, S. Giordani, ACS Nano, 5 (2011), 1198-1206. 